\title{
Fusobacterium naviforme
}

National Cancer Institute

\section{Source}

National Cancer Institute. Fusobacterium naviforme. NCI Thesaurus. Code C114252.

A species of obligately anaerobic, Gram negative, rod shaped bacteria assigned to the phylum Fusobacteria. This bacteria is non-spore forming, indole positive, nonfermentative, and characteristically produce butyrate as a major end product of its metabolism. F. naviforme has been isolated in human clinical samples and may cause infections in humans. 